\title{
DISTRIBUTION OF RESIDUES OF CERTAIN SECOND-ORDER LINEAR RECURRENCES MODULO $p$-II
}

\author{
Lawrence Somer \\ Catholic University of America, Washington, D.C. 20064 \\ (Submitted March 1989)
}

\section{Introduction}

Let $(u)=u(a, b)$, called the Lucas sequence of the first kind (LSFK), be a second-order linear recurrence satisfying the relation

$$
u_{n+2}=a u_{n+1}+b u_{n} \text {, }
$$

where $u_{0}=0, u_{1}=1$, and the parameters $a$ and $b$ are integers. Let $D=\alpha^{2}+4 b$ be the discriminant of $u(a, b)$. Let $(v)=v(a, b)$, called the Lucas sequence of the second kind (LSSK), be a recurrence satisfying (1) with initial terms $v_{0}=2, v_{1}=$ a. Throughout this paper, $p$ will denote an odd prime unless specified otherwise. Further, $d$ will always denote a residue modulo $p$. The period of $u(a, b)$ modulo $p$ will be denoted by $\mu(p)$. It is known (see [5]) that, if $p \nmid b$, then $u(a, b)$ is purely periodic modulo $p$. We will always assume that, in the LSFK $u(\alpha, b), p \nmid b$. The restricted period of $u(\alpha, b)$ modulo $p$, denoted by $\alpha(p)$, is the least positive integer $t$ such that $u_{n+t} \equiv s u_{n}(\bmod p)$ for all nonnegative integers $n$ and some nonzero residue $s$. Then $s$ is called the principal multiplier of $(u)$ modulo $p$. It is easy to see that $\alpha(p) \mid \mu(p)$ and that $\beta(p)=\mu(p) / \alpha(p)$ is the exponent of the principal multiplier $s$ of $(u)$ modulo $p$.

We will let $A(d)$ denote the number of times the residue $d$ appears in a full period of $u(a, b)$ modulo $p$ and $N(p)$ denote the number of distinct residues appearing in $u(a, b)$ modulo $p$. In a previous paper [13], the author considered the LSFK $u(\alpha, 1)$ modulo $p$ and gave constraints for the values which $A(d)$ can attain. In particular, it was shown that $A(d) \leq 4$ for all $d$. Upper and lower bounds for $N(p)$ were given in terms of $\alpha(p)$. Schinzel [8] improved on the constraints given in [13] for the values $A(d)$ can have in the LSFK $u(\alpha, 1)$ modulo $p$.

In this paper we will consider the LSFK $u(\alpha,-1)$ modulo $p$ and determine the possible values for $A(d)$. In particular, we will show that $A(d) \leq 2$ for all $d$. We will also obtain upper bounds for $N(p)$. If $\alpha(p)$ is known, we will determine $N(p)$ exactly. Schinzel [8] also presented results concerning $A(d)$ for the LSFK $u(\alpha,-1)(\bmod p)$, citing a preprint on which the present paper is based.

In [12], the author obtained the following partial results concerning $A(d)$ in the LSFK $u(\alpha,-1)(\bmod p)$.

Theorem 1: Consider the LSFK $u(\alpha,-1)$ modulo $p$ with discriminant $D=\alpha^{2}-4$.

(i) If $p \geq 5$ and $p \mid D$, then there exists a residue $d$ such that $A(d)=0$.

(ii) If $p \mid D$, then $A(d) \neq 0$ for any $d$. In particular, we must have that $\alpha \equiv \pm 2$ $(\bmod p)$. If $a \equiv 2(\bmod p)$, then

$u_{n} \equiv n(\bmod p)$

and $A(d)=1$ for all $d$. If $a \equiv-2(\bmod p)$, then

$u_{n} \equiv(-1)^{n+1} n(\bmod p)$

and $A(d)=2$ for all $d$. 


\section{Preliminaries}

A general multiplier of $u(a, b)(\bmod p)$ is any nonzero residue $s^{\prime}$ such that

$$
u_{n+t} \equiv s^{\prime} u_{n} \quad(\bmod p)
$$

for some fixed positive integer $t^{\prime}$ and all nonzero integers $n$. It is known that, if $s$ is the principal multiplier of $u(a, b)(\bmod p)$ and $s^{\prime}$ is a general multiplier of $u(a, b)(\bmod p)$, then

$$
s^{\prime} \equiv s^{i}(\bmod p)
$$

for some $i$ such that $0 \leq i \leq \beta(p)-1$.

For the LSFK $u(a, b)$, let $k=\alpha(p)$. We will let $A_{i}(d)$ denote the number of times the residue $d$ appears among the terms

$$
u_{k i}, u_{k i+1}, \ldots, u_{k i+k-1} \text { modulo } p \text {, }
$$

where $0 \leq i \leq \beta(p)-1$. Results concerning $A_{i}(d)$ will be obtained for the LSFK $u(\alpha,-1)(\bmod p)$.

The following results concerning $u(a, b)$ and $v(a, b)$ are well known:

$$
v_{n}^{2}-D u_{n}^{2}=4(-b)^{n}
$$

Proofs can be found in [4].

\section{The Main Theorems}

Our results concerning the distribution of residues in the LSFK $u(\alpha,-1)$ modulo $p$ will depend on knowledge of the values of $\alpha(p), \beta(p)$, and $(D / p)$, where $(D / p)$ denotes the Legendre symbol. Theorems 2 and 3 will provide information on the values $\mu(p), \alpha(p)$, and $\beta(p)$ can take for the LSFK $u(\alpha,-1)$ depending on whether $(D / p)=0,1$, or -1 .

Theorem 2: Let $u(a, b)$ be a LSFK. Then

$$
\alpha(p) \mid p-(D / p) \text {. }
$$

Further, if $p / D$, then

$$
\alpha(p) \mid(p-(D / p)) / 2
$$

if and only if $(-b / p)=1$. Moreover, if $(D / p)=1$, then

$$
\mu(p) \mid p-1
$$

Proof: Proofs of (4) and (6) are given in [4, pp. 44-45] and [1, pp. 315-17]. Proofs of (5) are given in [6, p. 441] and [1, pp. 318-19].

Theorem 3: Consider the LSFK $u(\alpha,-1)$ with discriminant $D$. Suppose that $p \not D$. Let $D^{\prime}$ be the square-free part of $D$. If $|\alpha| \geq 3$, let $\varepsilon$ be the funcamental unit of $Q\left(\sqrt{D^{\prime}}\right)$. Let $s$ be the principal multiplier of $u(a,-1)$ modulo $p$.

(i) $\beta(p)=1$ or $2 ; s \equiv 1$ or $-1(\bmod p)$.

(ii) If $\alpha(p) \equiv 0(\bmod 2)$, then $\beta(p)=2$.

(iii) If $\alpha(p) \equiv 1(\bmod 2)$, then $\beta(p)$ may be 1 or 2 .

(iv) If $(2-\alpha / p)=(2+\alpha / p)=-1$, then $\alpha(p) \equiv 0(\bmod 2)$ and $\beta(p)=2$

(v) If $(2-\alpha / p)=1$ and $(2+\alpha / p)=-1$, then $\alpha(p) \equiv 1(\bmod 2)$ and $\beta(p)=2$.

(vi) If $(2-\alpha / p)=-1$ and $(2+\alpha / p)=1$, then $\alpha(p) \equiv 1(\bmod 2)$ and $\beta(p)=1$.

(vii) If $p \equiv 1(\bmod 4),(D / p)=1$, and the norm of $\varepsilon$ is -1 , then $\alpha(p) \mid(p-1) / 4$.

Proof: This is proved in [11, pp. 328-31]. 
We are now ready for the statement of our principal theorems. Following the notation introduced by Schinzel in [8], we will let $S=S(p)$ denote the set of all the values which $A(d)$ attains in the LSFK $u(\alpha,-1)$ modulo $p$.

Theorem 4: Let $u(\alpha,-1)$ be an LSFK. Suppose that $\beta(p)=1$, and let $k=\alpha(p)$. Then $k \equiv 1(\bmod 2)$. Let $A_{0}^{\prime}(d)$ denote the number of times the residue $d$ appears among the terms $u_{0}, u_{1}, \ldots, u_{(k-1) / 2}$ modulo $p$. Let $A_{1}^{\prime}(d)$ denote the number of times the residue $d$ appears among the terms $u_{(k+1) / 2}, u_{(k+3) / 2}, \ldots, u_{k}$ modulo $p$.

(i) $A(d)=A(-d)$.

(ii) If $p \geq 5$, then $S=\{0,1\}$.

(iii) $A^{\prime}(d)=0$ or 1 for $i=0,1$.

(iv) $A_{0}^{\prime}(d)=A_{1}^{\prime}(-d)$.

Theorem 5: Let $u(\alpha,-1)$ be an LSFK. Suppose that $\alpha(p) \equiv 1(\bmod 2)$ and $\beta(p)=2$.

(i) $A(d)=A(-d)$.

(ii) If $p \geq 5$, then $S=\{0,2\}$.

(iii) If $d \neq 0(\bmod p)$, then $A_{i}(d)=0$ or 2 for $i=0,1$.

(iv) $A_{0}(0)=A_{1}(0)=1$.

(v) $A_{0}(d)=A_{1}(-d)$.

Theorem 6: Let $u(\alpha,-1)$ be an LSFK with discriminant $D$. Suppose $\alpha(p) \equiv 0$ (mod 2). Then $\beta(p)=2$ and $(-D / p)=1$.

(i) $A(d)=A(-d)$.

(ii) $A(d)=1$ if and only if $d \equiv \pm 2 / \sqrt{-D}(\bmod p)$.

(iii) If $p \geq 5$, then $S=\{0,1,2\}$.

(iv) If $d \not 0$ or $\pm 2 / \sqrt{-D}(\bmod p)$, then $A_{i}(d)=0$ or 2 for $i=0,1$.

(v) If $d \equiv 0$ or $\pm 2 / \sqrt{-D}(\bmod p)$, then $A_{i}(d)=1$ for $i=0,1$.

(vi) $A_{0}(d)=A_{1}(-d)$.

Theorem 7: Let $u(\alpha,-1)$ be an LSFK. Suppose that $p \not D$ and $\alpha \neq \equiv, 1$, or -1 (mod $p)$. Let $D^{\prime}$ be the square-free part of $D$. Let $\varepsilon$ be the fundamental unit of $Q\left(\sqrt{D^{\prime}}\right)$. Let $c_{1}=0$ if $\alpha(p) \equiv 1(\bmod 2)$ and $c_{1}=1$ if $\alpha(p) \equiv 0(\bmod 2)$.

(i) $N(p) \equiv 1(\bmod 2)$.

(ii) $N(p) \leq(p-(D / p)) / 2+c_{1}$.

(iii) If $p \equiv 1(\bmod 4),(D / p)=1$, and $\varepsilon$ has norm -1 , then

$N(p) \leq(p-1) / 4+c_{1}$.

(iv) $N(p)=\alpha(p)+c_{1}$.

\section{Necessary Lemmas}

The following lemmas will be needed for the proofs of Theorems 4-7.

Lemma 1: Let $u(a, b)$ be an LSFK. Let $s$ be the principal multiplier of (u) modulo $p$ and let $k=\alpha(p)$. Then

$$
u_{k-n} \equiv(-1)^{n+1} s u_{n} / b^{n}(\bmod p) \text {, }
$$

for $0 \leq n \leq k$. In particular, if $b \equiv-1(\bmod p)$, then

$$
u_{k-n} \equiv-s u_{n}(\bmod p) \text {, }
$$

for $0 \leq n \leq k$.

Proof: We proceed by induction. Clearly,

Also,

$$
\begin{aligned}
& u_{k-0} \equiv 0 \equiv(-1)^{0+1} s u_{0} / b^{0} \equiv 0 \equiv u_{0}(\bmod p) . \\
& u_{k-1} \equiv b^{-1}\left(u_{k+1}-a u_{k}\right) \equiv b^{-1}\left(s u_{1}-a \cdot 0\right) \equiv(-1)^{1+1} s u_{1} / b^{1}(\bmod p) .
\end{aligned}
$$


Now assume that

and

$$
u_{k-n} \equiv(-1)^{n+1} s u_{n} / b^{n}(\bmod p)
$$

Then

$$
\begin{aligned}
u_{k-(n+1)} & \equiv(-1)^{n+2} s u_{n+1} / b^{n+1}(\bmod p) . \\
u_{k-(n+2)} & \equiv b^{-1}\left(u_{k-n}-a u_{k}-(n+1)\right) \\
& \equiv b^{-1}(-1)^{n+1} s\left[\left(b u_{n} / b^{n+1}\right)+\left(a u_{n+1} / b^{n+1}\right)\right] \\
& =b^{-1}(-1)^{n+1} s\left(u_{n+2} / b^{n+1}\right) \equiv(-1)^{n+3} s u_{n+2} / b^{n+2}(\bmod p) .
\end{aligned}
$$

The result for $b \equiv-1(\bmod p)$ follows by inspection.

Lemma 2: Let $u(a, b)$ be an LSFK. Let $n$ and $c$ be positive integers such that $n+c \leq \alpha(p)-1$. Let $k=\alpha(p)$. Then

$$
\left(u_{n+c} / u_{n}\right)\left(u_{k-n} / u_{k-n-c}\right) \equiv(-b)^{c}(\bmod p) \text {. }
$$

Proof: This follows from congruence (7) in Lemma 1. Another proof is given in [12, p. 123].

Lemma 3: Consider the LSFK $u(a, b)$. Let $c$ be a fixed integer such that $1 \leq$ $c \leq \alpha(p)-1$. Then the ratios $u_{n+c} / u_{n}$ are all distinct modulo $p$ for $1 \leq n \leq$ $\alpha(p)-1$.

Proof: This is proved in [12, pp. 120-21].

Lemma 4: Let $u(\alpha,-1)$ be an LSFK and let $k=\alpha(p)$. Then

$$
u_{n} \equiv \pm u_{n+c}(\bmod p)
$$

for any positive integers $n$ and $c$ such that either $n+c \leq k / 2$ or it is the case that $n \geq k / 2$ and $n+c \leq k-1$.

Proof: Suppose there exist positive integers $n$ and $c$ such that $n+c \leq k-1$ and

Then

$$
u_{n} \equiv \pm u_{n+c}(\bmod p) \text {. }
$$

By Lemma 2,

$$
u_{n+c} / u_{n} \equiv \pm 1(\bmod p) \text {. }
$$

hence,

$$
\left(u_{n+c} / u_{n}\right)\left(u_{k-n} / u_{k-n-c}\right) \equiv 1^{c} \equiv 1(\bmod p) \text {; }
$$

$$
u_{k-n} / u_{k-n-c} \equiv u_{n+c} / u_{n} \equiv \pm 1(\bmod p) \text {. }
$$

Thus, by Lemma 3 ,

leading to

$$
n+c=k-n
$$$$
n=(k-c) / 2 \text {. }
$$

Consequent1y,

$$
n=(k-c) / 2 \text { and } n+c=(k+c) / 2 \text {. }
$$

The result now follows.

Lemma 5: Let $u(\alpha,-1)$ be an LSFK and let $k=\alpha(p)$. Let $N_{1}$ be the largest integer $t$ such that there exist integers $n_{1}, n_{2}, \ldots, n_{t}$ for which $1 \leq n_{i} \leq[k / 2]$ and $u_{n_{i}} \not \equiv \pm u_{n_{j}}(\bmod p)$ if $1 \leq i<j \leq[k / 2]$, where $[x]$ is the greatest integer less than or equal to $x$. Then

$$
N(p)=2 N_{1}+1
$$

Proof: By Theorem 3, $\beta(p)=1$ or 2 . First, suppose that $\beta(p)=2$. Then -1 is the principal multiplier of $(u)$ modulo $p$ and the residue $-d$ appears in $(u)$ 
DISTRIBUTION OF RESIDUES OF CERTAIN SECOND-ORDER LINEAR RECURRENCES MODULO $p$ - II

modulo $p$ if and only if $d$ appears in $(u)$ modulo $p$. Moreover, it follows from Lemma 1 and the fact that -1 is a principal multiplier of $(u)$ modulo $p$ that if $d \neq 0(\bmod p)$ and $d$ appears in $(u)(\bmod p)$, then $d \equiv \pm u_{n_{i}}(\bmod p)$ for some $i$ such that $1 \leq i \leq N_{l}$. Including the residue 0 , we see that (10) holds.

Now suppose that $\beta(p)=1$. By congruence (8) in Lemma 1 , the residue $-d$ appears in $(u)$ modulo $p$ if and only if $d$ appears in $(u)$ modulo $p$. It also follows from Lemma 1 that, if $d \not \equiv 0(\bmod p)$ and $d$ appears in $(u)$ modulo $p$, then $d \equiv \pm u_{n_{i}}(\bmod p)$ for some $i$ such that $1 \leq i \leq N_{1}$. Counting the residue 0 , we see that the result follows.

Lemma 6: Let $u(a,-1)$ be an LSFK. Let $k=\alpha(p)$. Let $A^{\prime}(d)$ denote the number of times the residue $d$ appears among the terms $n_{1}, n_{2}, \ldots, n_{[k / 2]}$ modulo $p$. Let $N_{1}$ be defined as in Lemma 5 .

(i) $A^{\prime}(d)+A^{\prime}(-d)=0$ or 1 .

(ii) $N_{1}=[k / 2]$.

Proof: (i) follows from Lemma 4; (ii) follows from (i).

Lemma 7: Let $u(a, b)$ be an LSFK. Suppose that $p \not b$. Let $s$ be the principal multiplier of $(u)$ modulo $p$ and $s^{j}$ be a general multiplier of $(u)$ (mod $\left.p\right)$, where $1 \leq j \leq \beta(p)-1$. Then

$$
A(d)=A\left(s^{j} d\right) \text {. }
$$

Proof: This is proved in [13].

Lemma 8: Let $u(\alpha,-1)$ be an LSFK with discriminant $D$. Suppose that $\alpha(p) \equiv 0$ $(\bmod 2)$. Let $k=\alpha(p)$. Then

$$
u_{k / 2} \equiv \pm 2 / \sqrt{-D}(\bmod p) \text {. }
$$

Proof: Since $\alpha(p) \equiv 0(\bmod 2)$, it follows from (4) that $p \not D$. By (2), it follows that

$$
v_{k / 2}^{2}-D u_{k / 2}^{2}=4(1)^{k / 2}=4 \text {. }
$$

Now, $u_{k} / 2 \not \equiv 0(\bmod p)$. Thus, by $(3), v_{k / 2} \equiv 0(\bmod p)$. Hence, by $(11)$, $-D u_{k / 2}^{2} \equiv 4(\bmod p)$

and the result follows.

\section{Proofs of the Main Theorems}

We are finally ready to prove Theorems 4-7.

Proof of Theorem 4: The fact that $\alpha(p) \equiv 1$ (mod 2) follows from Theorem 3.

(i) and (iv) follow from Lemma 1; (ii) follows from Theorem 1(i), Lemma 6(i), and Lemma 1; (iii) follows from Lemma 6 (i) and the fact that $A(0)=1$.

Proof of Theorem 5: (i) follows from Lemma 7; (ii) and (iii) follow from Theorem 1(i), Lemma 6(i), Lemma 1 , and the fact that -1 is the principal multiplier of $u(a,-1)$ modulo $p$; (iv) follows by inspection; and (v) follows from the fact that -1 is the principal multiplier of $(u)$ modulo $p$.

Proof of Theorem 6: The fact that $\beta(p)=2$ follows from Theorem 3. The fact that $(-D / p)=1$ follows from Lemma 8 .

(i) follows from Lemma 7; (ii), (iv), and (v) follow from Lemmas 8, 6(i), and 1 and the fact that -1 is the principal multiplier of ( $u$ ) modulo $p$; (iii) follows from Theorem 1(i), Lemma 6(i), Lemma 1 and the fact that -1 is the principal multiplier of $u(\alpha,-1)$ modulo $p$; and (vi) follows from the fact that -1 is the principal multiplier of $(u)$ modulo $p$. 
Remark: Note that Theorem 3 gives conditions for the hypotheses of Theorems 46 to be satisfied.

Proof of Theorem 7: (i) follows from Lemma 5; (ii) follows from Lemma 5, Lemma 6(ii), and Theorem 2; (iii) This follows from Lemma 5, Lemma 6(ii), and Theorem 3(vii); and (iv) follows from Lemmas 5 and 6(ii).

\section{Special Cases}

For completeness, we present Theorems 8 and 9 which detail special cases we have not treated thus far. For these theorems, $p$ will designate a prime, not necessarily odd.

Theorem 8: Let $u(\alpha,-1)$ be an LSFK. Suppose $p \nmid D$.

(i) If $\alpha \equiv 0(\bmod p)$, then $\alpha(p)=2, \beta(p)=2, N(p)=3, A(0)=2, A(1)=$ $A(-1)=1$, and $A(d)=0$ if $d \neq 0,1$, or $-1(\bmod p)$.

(ii) If $\alpha \equiv 1(\bmod p)$ and $p>2$, then $\alpha(p)=3, \beta(p)=2, N(p)=3, A(0)=$ $A(1)=A(-1)=2$, and $A(d)=0$ if $d \neq 0,1$, or $-1(\bmod p)$.

(iii) If $a \equiv 1(\bmod p)$ and $p=2$, then $\alpha(p)=3, \beta(p)=1, N(p)=2, A(0)=1$, and $A(1)=2$.

(iv) If $\alpha \equiv-1(\bmod p)$ and $p>2$, then $\alpha(p)=3, \beta(p)=1, N(p)=3, A(0)=$ $A(1)=A(-1)=1$, and $A(d)=0$ if $d \not \equiv 0,1$, or $-1(\bmod p)$.

Proof: (i)-(iv) follow by inspection.

Theorem 9: Let $u(\alpha,-1)$ be an LSFK. Suppose that $p \mid D$. Then $\alpha \equiv \pm 2(\bmod p)$. If $a \equiv 2(\bmod p)$, then $\alpha(p)=p, \beta(p)=1, N(p)=p$, and $A(d)=1$ for all residues $d$ modulo $p$. If $p>2$ and $a \equiv-2(\bmod p)$, then $\alpha(p)=p, \beta(p)=2, N(p)=$ $p$, and $A(d)=2$ for all residues $d$ modulo $p$.

Proof: This follows from Theorem 1(ii).

Remark: If $D \equiv 0(\bmod p)$, we see from Theorem 9 that the residues of $u(\alpha,-1)$ are equidistributed modulo $p$. See [7, p. 463] for a comprehensive list of references on equidistributed linear recurrences.

\section{Concluding Remarks}

In [8] and [13] it was shown that, for the LSFK $u(\alpha, 1)$ modulo $p, A(d) \leq 4$. In the present paper it was shown that, for the $\operatorname{LSFK} u(\alpha,-1)$ modulo $p, A(d) \leq$ 2. In [14] we extend these results considerably. Specifically, let $w(a, b)$ be a second-order linear recurrence with arbitrary initial terms $w_{0}$, $w_{1}$ over the finite field $F_{q}$ satisfying the relation

$$
w_{n+2}=a w_{n+1}+b w_{n} \text {. }
$$

where $b \neq 0$. Then

$$
A(d) \leq 2 \cdot \operatorname{ord}(-b)
$$

for all elements $d \in E_{q}$, where ord $(x)$ denotes the order of $x$ in $F_{q}$.

\section{$\underline{\text { References }}$}

1. R. P. Backstrom. "On the Determination of the Zeros of the Fibonacci Sequence." Fibonacci Quarterly 4.4 (1966):313-22.

2. G. Bruckner. "Fibonacci Sequences Modulo a Prime $p \equiv 3(\bmod 4)$ " Fibonacci Quarterly 8.2 (1970):217-20.

3. S. A. Burr. "On Moduli for Which the Fibonacci Sequence Contains a Complete System of Residues." Fibonacci Quarterly 9.4 (1971):497-504. 
4. R. D. Carmichael. "On the Numerical Factors of the Arithmetic Forms $\alpha^{n} \pm$ $\beta^{n}$." Ann. Math. Second Series 15 (1913):30-70.

5. R. D. Carmichael. "On Sequences of Integers Defined by Recurrence Relations." Quart. J. Pure Appl. Math. 48 (1920):343-72.

6. D. H. Lehmer. "An Extended Theory of Lucas' Functions." Ann. Math. Second Series 31 (1930):419-48.

7. R. Lidl \& H. Niederreiter. Finite Fields. Reading, Mass.: Addison-Wesley, 1983.

8. A. Schinze1. "Special Lucas Sequences, Including the Fibonacci Sequence, Modulo a Prime." To appear.

9. A. P. Shah. "Fibonacci Sequences Modulo m." Fibonacei Quarterly 6.1 (1968): $139-41$.

10. L. Somer. "The Fibonacci Ratios $F_{k+1} / F_{k}$ Modulo $p$." Fibonacci Quarterly 13. 4 (1975):322-24.

11. L. Somer. "The Divisibility Properties of Primary Lucas Recurrences with Respect to Primes." Fibonacci Quarterly 18.4 (1980):316-34.

12. L. Somer. "Primes Having an Incomplete System of Residues for a Class of Second-Order Linear Recurrences." Applications of Fibonacci Numbers. Ed. by A. N. Philippou, A. F. Horadam, \& G. E. Bergum. Dordrecht, Holland: Kluwer Academic Publishers, 1988, pp. 113-41.

13. L. Somer. "Distribution of Residues of Certain Second-Order Linear Recurrences Modulo p." Applications of Fibonacci Numbers, Vol. 3. Ed. G. E. Bergum, A. N. Philippou, and A. F. Horadam. Dordrecht, Holland: Kluwer Academic Publishers, 1990, pp. 311-24.

14. L. Somer, H. Niederreiter, * A. Schinze1. "Maximal Frequencies of Elements in Second-Order Recurrences Over a Finite Field." To appear. 